\title{
Nonlinear measures of association with kernel canonical correlation analysis and applications
}

\author{
Su-Yun Huang ${ }^{\mathrm{a}, *}$, Mei-Hsien Lee ${ }^{\mathrm{b}}$, Chuhsing Kate Hsiao ${ }^{\mathrm{c}}$ \\ anstitute of Statistical Science, Academia Sinica, Taipei 11529, Taiwan, ROC \\ ${ }^{\mathrm{b}}$ Department of Mathematics and Computer Science Education, Taipei Municipal University of Education, Taiwan, ROC \\ ${ }^{c}$ Department of Public Health and Institute of Epidemiology, National Taiwan University, Taiwan, ROC
}

\section{A RTICLE IN F O}

\section{Article history:}

Received 7 December 2007

Received in revised form

7 October 2008

Accepted 8 October 2008

Available online 1 November 2008

\section{Keywords:}

Association measure

Canonical correlation analysis

Dimension reduction

Kernel method

Multivariate analysis

Reproducing kernel

Reproducing kernel Hilbert space

Test of independence

\begin{abstract}
A B S T R A C T
Measures of association between two sets of random variables have long been of interest to statisticians. The classical canonical correlation analysis (LCCA) can characterize, but also is limited to, linear association. This article introduces a nonlinear and nonparametric kernel method for association study and proposes a new independence test for two sets of variables. This nonlinear kernel canonical correlation analysis (KCCA) can also be applied to the nonlinear discriminant analysis. Implementation issues are discussed. We place the implementation of KCCA in the framework of classical LCCA via a sequence of independent systems in the kernel associated Hilbert spaces. Such a placement provides an easy way to carry out the KCCA. Numerical experiments and comparison with other nonparametric methods are presented.
\end{abstract}

(c) 2007 Elsevier B.V. All rights reserved.

\section{Introduction}

The description and measure of relation between two sets of variables have been of interests to many researchers. Hotelling (1936) introduced the notion of canonical correlation analysis to describe the linear relation (abbreviated as LCCA) between two sets. The LCCA is concerned with the linear relationship and aims to define a new orthogonal coordinate system for each of the two sets so that the new pair of coordinate systems are optimal in maximizing the correlations. The new systems of coordinates are linear systems of the original ones. Thus, the LCCA can only be used to describe linear relationship. In addition, via such linear relationship it extracts only linear features for linear dimension reduction. Another application of LCCA is to test stochastic independence between two sets of variables, under Gaussian assumptions. However, properties of the LCCA test become invalid, if data are not Gaussian, or not elliptically symmetrically distributed. In such case, one has to resort to some nonlinear and nonparametric strategy.

Motivated from the active development of statistical learning theory (Vapnik, 1998; Hastie et al., 2001; and references therein) and the popular and successful usage of various kernel machines (Cortes and Vapnik, 1995; Cristianini and Shawe-Taylor, 2000; Schölkopf and Smola, 2002), there has emerged a hybrid approach of the LCCA with a kernel machine (Akaho, 2001; Bach and Jordan, 2002), named kernel canonical correlation analysis (KCCA). The KCCA has also been studied recently by Gretton et al. (2005). All the above works are essentially on the computer science side. Not enough about its statistical properties are discussed. In statistical literature there has also been a wide concern and advanced development of the nonlinear canonical

\footnotetext{
* Corresponding author. Tel.: +8862 27835611; fax: +886227831523.

E-mail address: syhuang@stat.sinica.edu.tw (S.-Y. Huang).
} 
correlation analysis (NCCA), e.g., Dauxois et al. (1993, 2004), Dauxois and Nkiet (1997, 1998, 2002), Ramsay and Silverman (2005, Chapter 11), Hsing et al. (2005), Eubank and Hsing (2006), among many others.

In this article we focus on the study of kernel-based nonlinear association in the framework of a kernel generated Hilbert space and derive a simple implementation algorithm. We next utilize this approach to the problems of dimension reduction (features extraction) and test of independence. The rest of the article is organized as follows. In Section 2 we briefly review the classical LCCA and then introduce its kernelized companion KCCA in the framework of a reproducing kernel Hilbert space (RKHS). In Section 3 we discuss some issues concerning the implementation of KCCA by placing it in the framework of the classical LCCA. In Section 4 we introduce some nonlinear association measures and propose a new test of independence. We also provide some numerical experiments and a comparison study with other nonparametric methods including the rank-based correlation coefficients and spline-based independence test. An example using KCCA as a dimension reduction tool for discriminant purpose is also given in this section. Finally, concluding remarks and discussions are given in Section 5. Technical details and theoretical justification are given in Appendix A.

\section{Canonical correlation analysis: linear and kernel}

\subsection{Review of linear canonical correlation analysis}

Suppose the random vector $X$ of $q$ components has a probability measure $P$ on $\mathscr{X} \subset \mathfrak{R}^{q}$. It is partitioned into $q_{1}$ and $q_{2}$ components and, similarly, the covariance matrix is partitioned accordingly:

$$
X=\left[\begin{array}{l}
X^{(1)} \\
X^{(2)}
\end{array}\right], \quad \Sigma=\left[\begin{array}{ll}
\Sigma_{11} & \Sigma_{12} \\
\Sigma_{21} & \Sigma_{22}
\end{array}\right] .
$$

We are interested in finding the relation between $X^{(1)}$ and $X^{(2)}$. The LCCA describes the linear relation by reducing the correlation structure to the simplest possible form, i.e., a diagonal form. For the first pair of canonical variates, the LCCA seeks vectors $\alpha \in \mathfrak{R}^{q_{1}}$ and $\beta \in \mathfrak{R}^{q_{2}}$ to form linear variates $\alpha^{\prime} X^{(1)}$ and $\beta^{\prime} X^{(2)}$ that maximize the correlation, namely, it solves the following optimization problem:

$$
\rho_{1}:=\max _{[\alpha ; \beta] \in \Re^{q_{1}+q_{2}}} \alpha^{\prime} \Sigma_{12} \beta \quad \text { subject to } \alpha^{\prime} \Sigma_{11} \alpha=1 \text { and } \beta^{\prime} \Sigma_{22} \beta=1 \text {. }
$$

Denote the resulting vectors of (1) by $\left[\alpha_{1} ; \beta_{1}\right]$. The pair $\alpha_{1}$ and $\beta_{1}$ are called the first pair of linear canonical vectors, and the associated variates $\alpha_{1}^{\prime} X^{(1)}$ and $\beta_{1}^{\prime} X^{(2)}$ are called the first pair of linear canonical variates. For the rest pairs of canonical vectors and variates, it solves sequentially the same problem (1) with extra constraints of sequential orthonormality, i.e., for the $k$ th pair the extra constraints are

$$
\alpha_{k}^{\prime} \Sigma_{11} \alpha_{i}=0 \quad \text { and } \quad \beta_{k}^{\prime} \Sigma_{22} \beta_{i}=0, \quad \forall i=1, \ldots, k-1
$$

The optimization problem defined by (1) and (2) can be combined into

$$
\max _{A \in \Re^{q_{1} \times m}, B \in \Re^{q_{2} \times m}} \operatorname{trace}\left\{A^{\prime} \Sigma_{12} B\right\} \quad \text { subject to } A^{\prime} \Sigma_{11} A=I_{m} \text { and } B^{\prime} \sum_{22} B=I_{m},
$$

where $m=\min \left(q_{1}, q_{2}\right), A=\left[\alpha_{1}, \ldots, \alpha_{m}\right]$ and $B=\left[\beta_{1}, \ldots, \beta_{m}\right]$. The sets, $\left\{\alpha_{i}\right\}_{i=1}^{m}$ and $\left\{\beta_{i}\right\}_{i=1}^{m}$, are the leading coordinate axes and can be supplemented to form a pair of new coordinate systems for $\mathscr{X}_{1} \subset \mathfrak{R}^{q_{1}}$ and $\mathscr{X}_{2} \subset \mathfrak{R}^{q_{2}}$, respectively. The sequence of correlation coefficients $\left\{\rho_{1}, \rho_{2} \ldots, \rho_{m}\right\}$ describes only the linear relation between $X^{(1)}$ and $X^{(2)}$. The LCCA can be justified by assuming that $X^{(1)}$ and $X^{(2)}$ have a joint Gaussian distribution, and the likelihood ratio criterion for testing independence of $X^{(1)}$ and $X^{(2)}$ can be expressed entirely in terms of sample correlation coefficients, $\left\{\hat{\rho}_{1}, \hat{\rho}_{2} \ldots, \hat{\rho}_{m}\right\}$, based on a given data set. Assume that we have a data set $\left\{x_{j}\right\}_{j=1}^{n}$, where $x_{j}=\left[x_{j}^{(1)} ; x_{j}^{(2)}\right]$ is partitioned according to $\mathscr{X}_{=} \mathscr{X}_{1} \oplus \mathscr{X}_{2}$. Let $\mathbb{X}$ denote the data design matrix

$$
\mathbb{X}:=\left[\begin{array}{cc}
x_{1}^{(1)^{\prime}} & x_{1}^{(2)^{\prime}} \\
\vdots & \vdots \\
x_{n}^{(1)^{\prime}} & x_{n}^{(2)^{\prime}}
\end{array}\right]_{n \times\left(q_{1}+q_{2}\right)}:=\left[\mathbb{X}^{(1)}, \mathbb{X}^{(2)}\right]
$$

The sampling-based LCCA for $X^{(1)}$ and $X^{(2)}$ is to work on $\left[\mathbb{X}^{(1)}, \mathbb{X}^{(2)}\right]$. The covariance matrices in optimization problems (1) and (2) are replaced by their sample versions.

\subsection{Kernel generalization of canonical correlation analysis}

There are cases where linear correlations may not be adequate for describing association between $X^{(1)}$ and $X^{(2)}$. A natural alternative, therefore, is to explore and exploit their nonlinear relation. Several authors in the machine learning community 
(see Introduction) have resorted to kernel methods, which can provide a convenient way for nonlinear generalization of LCCA. Our steps of kernel generalization described below are different from the procedures in the above-mentioned literature. Our procedure is to work directly on the kernel generated Hilbert space instead of the kernel spectrum-based feature space and, for algorithmic implementation, our procedure makes use of the parametric notion of LCCA in the underlying RKHS. That is, our working procedure is simply the LCCA steps on kernel data, so that standard LCCA software can be applied. The extra computing efforts compared with the classical LCCA is to generate the kernel data. Technical details and theoretical justification are placed in Appendix A. Below we only give steps of our KCCA working procedure. Let

$$
\mathbb{K}^{(1)}=\kappa_{1}\left(\mathbb{X}^{(1)}, \mathbb{X}^{(1)}\right) \text { and } \mathbb{K}^{(2)}=\kappa_{2}\left(\mathbb{X}^{(2)}, \mathbb{X}^{(2)}\right) \text {, }
$$

where $\kappa_{1}$ and $\kappa_{2}$ are two positive definite kernel functions defined on $\mathscr{X}_{1} \times \mathscr{X}_{1}$ and $\mathscr{X}_{2} \times \mathscr{X}_{2}$, respectively, and $\mathbb{K}^{(i)}$ is the matrix whose $\left(j, j^{\prime}\right)$ th entry is given by $\kappa_{i}\left(x_{j}^{(i)}, x_{j^{\prime}}^{(i)}\right)$ for $i=1,2$, and $j, j^{\prime}=1, \ldots, n$. The KCCA works by carrying out the classical LCCA procedure on the kernel data $\left[\mathbb{K}^{(1)}, \mathbb{K}^{(2)}\right]$. In summary, the KCCA procedure consists of two major steps:

(a) Transform the data points to a kernel representation as in (4) and obtain kernel matrices $\mathbb{K}^{(1)}$ and $\mathbb{K}^{(2)}$. Note that the kernel matrices $\mathbb{K}^{(1)}$ and $\mathbb{K}^{(2)}$ often have effective ranks much lower than their sizes. Thus, some sort of regularization is necessary to avoid the numerical instability incurred in canonical analysis. At this moment, tentatively assume that regularized kernel matrices $\left[\tilde{\mathbb{K}}^{(1)}, \tilde{\mathbb{K}}^{(2)}\right]$ are given. Discussion concerning their derivation is deferred to Section 3.

(b) The KCCA is simply the classical LCCA on regularized kernel data $\tilde{\mathbb{K}}^{(1)}$ and $\tilde{\mathbb{K}}^{(2)}$. Let $M_{i j}=\operatorname{cov}\left\{\tilde{\mathbb{K}}^{(i)}, \tilde{\mathbb{K}}^{(j)}\right\}$ for $i, j=1,2$. That is, KCCA solves the generalized singular value decomposition (SVD) of $M_{12}$ as $U A V^{\prime}$, where $U$ and $V$ are two orthogonal matrices in the metrics $U^{\prime} M_{11} U=I$ and $V^{\prime} M_{22} V=I$, respectively, and $\Lambda$ is a diagonal matrix consisting of canonical correlation coefficients.

\section{Implementation of KCCA}

There have been various implementation algorithms for KCCA (e.g., Gretton et al., 2003, 2005; Hardoon et al., 2004; Kuss and Graepel, 2003). The classical LCCA is a long and well developed data analysis tool and has been implemented in many statistical and mathematical software (e.g., canoncorr in Matlab; cancor in R; cancor in Splus; and proc cancorr in SAS). As pointed out earlier, the working procedure of KCCA can be carried out as LCCA on regularized kernel data. The extra effort required is to prepare the data in an appropriate kernel form. This is a crucial step for efficient computation and for accurate estimation of the nonlinear canonical correlation structure. After regularized kernel data are generated, next they are fed into an LCCA package. In all our numerical experiments, we have used the Matlab m-file canoncorr for the canonical correlation analysis. Our implementation discussion below will focus on kernel data preparation including regularization and parameter selection. One main advantage of using a conventional LCCA code is to allow statisticians or practitioners unfamiliar with kernel machines to have an easy access to implementing KCCA and its applications to statistical data analysis and inference. The regularization step is adopted to avoid numerical instability and to enhance the computational efficiency. The parameter selection is to find an appropriate kernel representation so that data in the associated RKHS have an approximately elliptical symmetric distribution.

\subsection{Regularization}

Recall the optimization problem of LCCA on kernel data $\mathbb{K}^{(1)}$ and $\mathbb{K}^{(2)}$. It is a generalized SVD of $\operatorname{cov}\left\{\mathbb{K}^{(1)}\right.$, $\left.\mathbb{K}^{(2)}\right\}$ with respect to metrics by $\operatorname{cov}\left\{\mathbb{K}^{(1)}\right\}$ and $\operatorname{cov}\left\{\mathbb{K}^{(2)}\right\}$. Note that the sample covariance matrices based on kernel data $\mathbb{K}^{(1)}$ and $\mathbb{R} \mathbb{K}^{(2)}$ are singular and have effective ranks much lower than their sizes. The optimization problem is ill-conditioned and some sort of regularization is needed. One commonly used regularization method is the ridge-type regularization:

The ridge-type regularization adds a small quantity to the diagonals,

$$
A^{\prime}\left(\operatorname{cov}\left\{\mathbb{K}^{(1)}\right\}+\lambda_{1} I_{n}\right) A=I_{m} \quad \text { and } \quad B^{\prime}\left(\operatorname{cov}\left\{\mathbb{K}^{(2)}\right\}+\lambda_{2} I_{n}\right) B=I_{m}
$$

to stabilize the numerical computation for solving the associated generalized SVD. This approach has been adopted in, e.g., Gretton et al. (2003, 2005), Hardoon et al. (2004), Kuss and Graepel (2003). Although this ridge-type regularization lessens the numerical instability, it does not solve the problem of inferior estimation. Due to several reasons, we will not adopt such a ridge-type regularization. A more appropriate way to deal with the problem is to find a reduced-column approximation to $\mathbb{K}^{(i)}$, denoted by $\tilde{\mathbb{K}}^{(i)}$, so that $\tilde{\mathbb{K}}^{(i)}$ has full column rank and its column space, denoted by $\mathscr{C}\left(\tilde{\mathbb{K}}^{(i)}\right)$, provides a good approximation to $\mathscr{C}(\mathbb{K})$. This approximation will enhance the numerical stability without much information loss. Here we introduce three approaches for reduced-column approximation.

Reduced-column approximation by optimal basis subset. Let $\mathbb{K}^{(1)}$ and $\mathbb{K}^{(2)}$ be the $n \times n$ kernel matrices, where $n$ is the data size. The SVD factors and then extracts the leading right singular vectors from $\mathbb{K}^{(1)}$ and $\mathbb{K}^{(2)}$ :

$$
\mathbb{K}^{(1)}=\mathbb{U}^{(1)} \Lambda_{1} \mathbb{U}^{(1)^{\prime}} \text { and } \mathbb{K}^{(2)}=\mathbb{U}^{(2)} \Lambda_{2} \mathbb{U}^{(2)^{\prime}} \text {. }
$$


Let the leading columns be denoted by $\tilde{\mathbb{U}}^{(1)}$ and $\tilde{\mathbb{U}}^{(2)}$ with the column sizes $m_{1}$ and $m_{2}$, respectively. Next, columns of $\mathbb{K}^{(1)}$ and $\mathbb{K}^{(2)}$ are projected onto the column spaces of $\tilde{\mathbb{U}}^{(1)}$ and $\tilde{\mathbb{U}}^{(2)}$ and the reduced-column approximation of kernel data are given by

$$
\tilde{\mathbb{K}}^{(1)}=\mathbb{K}^{(1)^{\prime}} \tilde{U}^{(1)} \text { and } \tilde{\mathbb{K}}^{(2)}=\mathbb{K}^{(2)^{\prime}} \tilde{\mathbb{U}}^{(2)} \text {. }
$$

The classical LCCA is then acting on the reduced kernel matrix $\tilde{\mathbb{K}}=\left[\tilde{\mathbb{K}}^{(1)}, \tilde{\mathbb{K}}^{(2)}\right]$ instead of the full kernel matrix $\left[\mathbb{K}^{(1)}, \mathbb{K}^{(2)}\right]$. This SVD approach to extracting right singular vectors is a reduced-column approximation by optimal basis subset.

Reduced-column approximation by random basis subset. Let $\mathbb{X}^{(1)}$ and $\mathbb{X}^{(2)}$ denote the full sets of partitioned data, and let $\tilde{\mathbb{X}}^{(1)}$ and $\tilde{\mathbb{X}}^{(2)}$ be subsets of the full sets, i.e., $\tilde{\mathbb{X}}^{(i)}=\left\{\tilde{x}_{j}^{(i)}\right\}_{j=1}^{m_{i}} \subset\left\{x_{j}^{(i)}\right\}_{j=1}^{n}$ for $i=1,2$. Data designs, $\mathbb{X}^{(i)}$ and $\tilde{\mathbb{X}}^{(i)}$, are arranged as observations by rows and variables by columns. The subset method selects small portion subsets from the full sets to form reduced-column kernel matrices

$$
\tilde{\mathbb{K}}^{(1)}=\kappa_{1}\left(\mathbb{X}^{(1)}, \tilde{\mathbb{X}}^{(1)}\right) \text { and } \tilde{\mathbb{K}}^{(2)}=\kappa_{2}\left(\mathbb{X}^{(2)}, \tilde{\mathbb{X}}^{(2)}\right) \text {, }
$$

where $\kappa_{1}\left(\mathbb{X}^{(1)}, \tilde{\mathbb{X}}^{(1)}\right)=\left[\kappa_{1}\left(x_{i}^{(1)}, \tilde{x}_{j}^{(1)}\right)\right]$ with $x_{i}^{(1)}$ from $\mathbb{X}^{(1)}$ and $\tilde{x}_{j}^{(1)}$ from $\tilde{\mathbb{X}}^{(1)}$. The reduced kernel $\tilde{\mathbb{K}}^{(1)}$ is a thin column matrix of size $n \times m_{1}$ with $m_{1}$ the row size of $\tilde{\mathbb{X}}^{(1)}$. The reduced kernel $\tilde{\mathbb{K}}^{(2)}$ is similarly defined. The subset size is often much smaller than the full set size, i.e., $m_{1}, m_{2} \ll n$. The choice of subsets $\tilde{\mathbb{X}}^{(1)}$ and $\tilde{\mathbb{X}}^{(2)}$ is often made by (stratified) uniform random sampling from the full sets $\mathbb{X}^{(1)}$ and $\mathbb{X}^{(2)}$. This approach is termed "random subset" or "random basis subset" and is the most economic one among the three regularization methods, ridge, optimal and random subset, in terms of computational complexity. The random subset method leads to a sparse representation with only a small number of kernel functions for the underlying model. This approach can effectively speed up the computation and can cut down the model complexity (Lee and Mangasarian, 2001; Williams and Seeger, 2001; Lee and Huang, 2007). Furthermore, as the subset selection is purely random, it is recommended for median to large data sets and is particularly useful for large data sets. Of course, one can always do better than pure randomness in subset selection at the expense of computing time. For example, SVD is an optimal basis approach. Snelson and Ghahramani (2005) and Wang et al. (2005) have proposed nice ways of training the reduced set selection. Other sequential adaptive algorithm for basis selection is also available (Smola and Schölkopf, 2000). All these methods to train for basis selection are more time consuming and require some search algorithms. The uniform random subset is a simple and economic alternative. However, there is always a trade-off between model bias and computational cost. Our suggestion is, for small sized problems, the SVD provides an optimal basis subset, which leads to a better approximation than various complicated subset training methods. As the data size $n$ becomes large, the random subset approach is recommended for its simplicity and being economic. A hybrid of random subset and SVD can be adopted as well, which is to use the SVD within a certain sized random subset.

A hybrid approach starts with an adequate proportion of kernel basis by random subset and then cuts down the effective dimensionality by the SVD approach (6).

\subsection{Parameter selection}

There are three parameters involved in the entire procedure of KCCA. One is the choice of kernel, the second is the kernel window width and the third is the regularization parameter (the ridge parameter, the number of singular vectors and the reduced set size). Throughout this article we use Gaussian kernel as our choice of kernel and the window width is set to $\sqrt{10 S_{\ell}}$ coordinatewise with $S_{\ell}$ the one-dimensional sample variance for $\ell=1, \ldots, q$. Such a choice of window width is based on our empirical experience and it provides a universal rule of thumb suggestion. Though it might not be optimal or even far from being optimal, it gives robust and satisfactory results. For a better parameter setting, a grid search over a certain appropriate search range can be done with the cost of computing time. More advanced search scheme based on uniform designs can be found in Huang et al. (2007). As for choices of reduced set size and the number of singular vectors, we suggest users to start with an adequate proportion of kernel basis and then cut down the effective dimensionality by the SVD approach (i.e., the hybrid approach) depending on the availability and performance of computer facility. As we do not use the ridge-type regularization here, we do not make any suggestion for it, but one can always resort to cross-validation, grid search, or simply by hand-pick.

\section{Measures of association and test of independence}

\subsection{Measures of association}

Like the LCCA, the KCCA leads to a sequence of correlation coefficients $\left\{\rho_{1}^{\kappa}, \rho_{2}^{\kappa}, \ldots\right\}$ arranged in descending order. The superscript $\kappa$ indicates that these coefficients are kernelized canonical correlation coefficients. Several measures of association in the literature are constructed as functions of the correlation coefficients derived from either LCCA (see, e.g., Jensen and Mayer, 1977; Anderson, 2003) or NCCA (Dauxois and Nkiet, 1998). Here we introduce three particular and commonly seen association measures. One is 
the maximal correlation and its kernelized version

$$
r_{\max }\left(X^{(1)}, X^{(2)}\right):=\rho_{1}, r_{\max }^{\kappa}\left(\tilde{\mathbb{K}}^{(1)}, \tilde{\mathbb{K}}^{(2)}\right):=\rho_{1}^{\kappa},
$$

one is the log-likelihood-ratio-based association measure and kernelization

$$
r_{\log }\left(X^{(1)}, X^{(2)}\right):=-\sum_{v} \log \left(1-\rho_{v}^{2}\right), \quad r_{\log }^{\kappa}\left(\tilde{\mathbb{K}}^{(1)}, \tilde{\mathbb{K}}^{(2)}\right):=-\sum_{v} \log \left(1-\left(\rho_{v}^{\kappa}\right)^{2}\right),
$$

and the other is

$$
r_{\mathrm{D} \& \mathrm{~N}}^{\mathrm{spl}}:=\frac{\sum_{v}\left(\rho_{v}^{\mathrm{spl}}\right)^{2}}{1+\sum_{v}\left(\rho_{v}^{\mathrm{spl}}\right)^{2}}
$$

by Dauxois and Nkiet using spline basis. Or, sometimes, we simply make direct use of the raw kernel correlation coefficients $\rho^{\kappa}$ 's.

Below we give some examples of the usage of these nonlinear association measures. In Example 1 we illustrate the nonlinear association measure using (8) and compare it with the classical Pearson correlation coefficient and the rank-based Kendall $\tau$ and Spearman $\rho$. In Example 11 we demonstrate the nonlinear association between two sets of multivariate data using the first two leading kernel correlation coefficients $\rho_{1}^{\kappa}$ and $\rho_{2}^{\kappa}$ and plot the data scatters of the first two kernel canonical variates. In Example 3 we demonstrate the use of leading kernel canonical variates for discriminant purpose. In Example 4 we use (9) to device a test of stochastic independence and compare it with Dauxois and Nkiet's (10).

Example 1 (Synthetic data set: association for two random variables). In this example, we use the first kernel canonical correlation coefficient in (8) as our association measure and compare it with the Pearson (linear) correlation coefficient and two well-known nonparametric rank-based association measures, Kendall $\tau$ and Spearman $\rho$. In the following three cases, let $X^{(1)}$ and $\varepsilon$ be two independent standard normals, and the models of $X^{(2)}$ considered are:

I. $X^{(2)}=f\left(X^{(1)}\right)+\varepsilon / k$ with $f(x)=\cos (\pi x)$;

II. $X^{(2)}=f\left(X^{(1)}\right)+\varepsilon / k$ with $f(x)=\exp (-|x|)$ and

III. $X^{(2)}=X^{(1)}+\varepsilon / k$;

where $k$ is a constant in $(0,10]$. In these three models, the amount of association increases with respect to $k$. In each setting, we generate 500 sample points of $X^{(1)}$ and $\varepsilon$, and take account of relationship between $X^{(1)}$ and $X^{(2)}$. Kernel data are generated using Gaussian kernel with the rule-of-thumb window width suggested in Section 3. Optimal basis by SVD (6) is used, and the sizes of basis sets $m_{1}$ and $m_{2}$ are so determined that the extracted optimal bases make up 99\% of eigenvalues of $\Lambda_{1}$ and $\Lambda_{2}$ in (5). Models I and II are nonlinear association models. Five quantities of association measures are considered and the corresponding curves are plotted in Fig. 1(a) and (b) for Models I and II, respectively. The solid curve in each plot represents the Pearson correlation of $X^{(2)}$ and $f\left(X^{(1)}\right)$ taken as the true target association measure between $X^{(1)}$ and $X^{(2)}$. The kernel correlation curve in circle well catches the true correlation curve. All the rest curves, Kendall $\tau$, Spearman $\rho$ and Pearson linear correlation, are around zero due to the symmetric structure of the functions $\cos (\pi x)$ and $\exp (-|x|)$. Overall speaking, kernel canonical correlation outperforms the Kendall $\tau$, Spearman $\rho$ and Pearson linear correlation and it follows the target correlation curve well in Models I and II. As for the linearly correlated case (Model III in Fig. 1(c)), all the correlation curves follow very closely the true curve except for Kendall $\tau$, which is a bit low from the true curve. Particularly note that the kernel canonical correlation curve follows the true curve well. It indicates that the nonlinear kernel method also works well for a linear association model.

Example 2 (Synthetic data set: association for two sets of variables). Let $X_{1}$ and $X_{2}$ be independent and identically distributed random variables having uniform distribution over the interval $(-2,2)$. Let

$$
Y_{1}=X_{1}^{2}+0.1 \varepsilon_{1} \text { and } Y_{2}=\cos \left(\pi X_{2}\right)+0.1 \varepsilon_{2} \text {, }
$$

where $\varepsilon$ 's are standard normal random noises. Let $X^{(1)}=\left(X_{1}, X_{2}\right)$ and $X^{(2)}=\left(Y_{1}, Y_{2}\right)$. In each simulation, we sample 1000 pairs of $\left(X^{(1)}, X^{(2)}\right)$ from the described model and calculate two leading pairs of sample canonical correlation coefficients and variates using both the linear and kernel CCA. In contrast to the optimal basis by SVD in Example 1 for regularizing kernel data, here we use a reduced kernel by random subset of size 100 as our choice of regularization. Of course, one can use the SVD approach, which gives the optimal basis and is better accurate for KCCA estimates. However, for a demonstrative purpose, here we choose the computationally economic random subset approach. The mutual correlation carried in the two leading pairs of canonical variates found by LCCA and KCCA are listed in Table 1 for 30 replicate runs. Reported are the average correlation coefficients and their standard errors. It is evident that kernel canonical variates capture more association measure between the two sets of variables. We randomly choose one copy from the 30 replicate runs and plot these 1000 data points along the two leading pairs of sample canonical variates. Fig. 2(a) is the data scatter of the first pair of linear canonical variates and Fig. 2(b) is the data scatter for the second pair of linear canonical variates. There is indication of some strong association left unexplained. Fig. 2(c) and (d) 
a

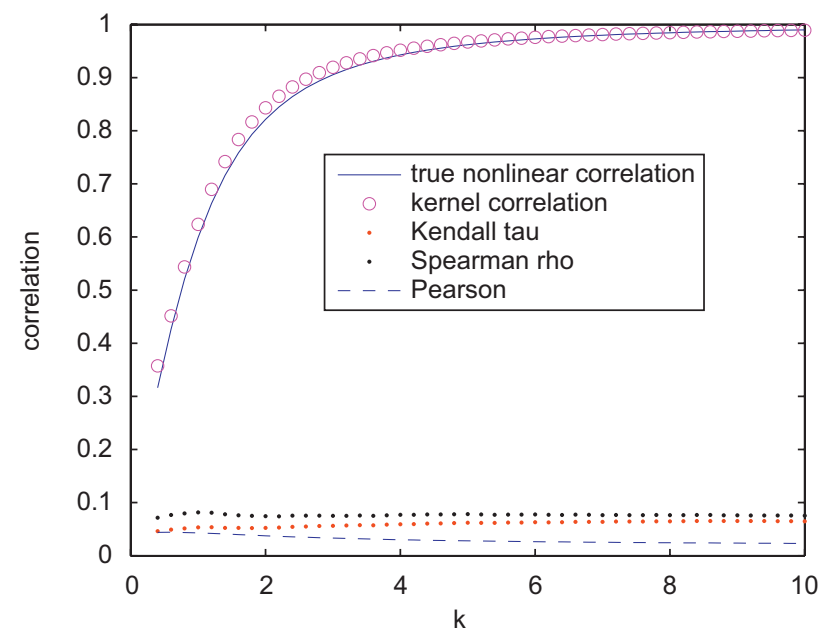

b

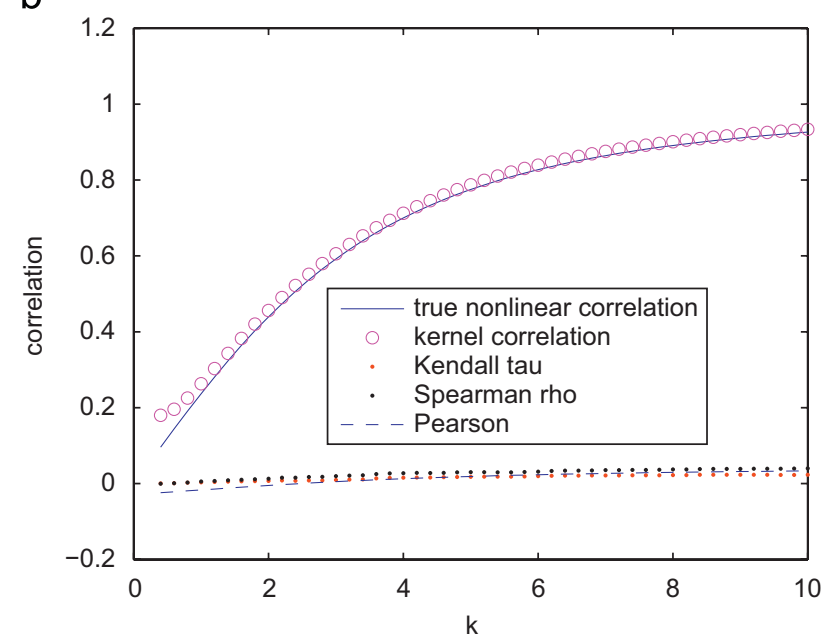

C

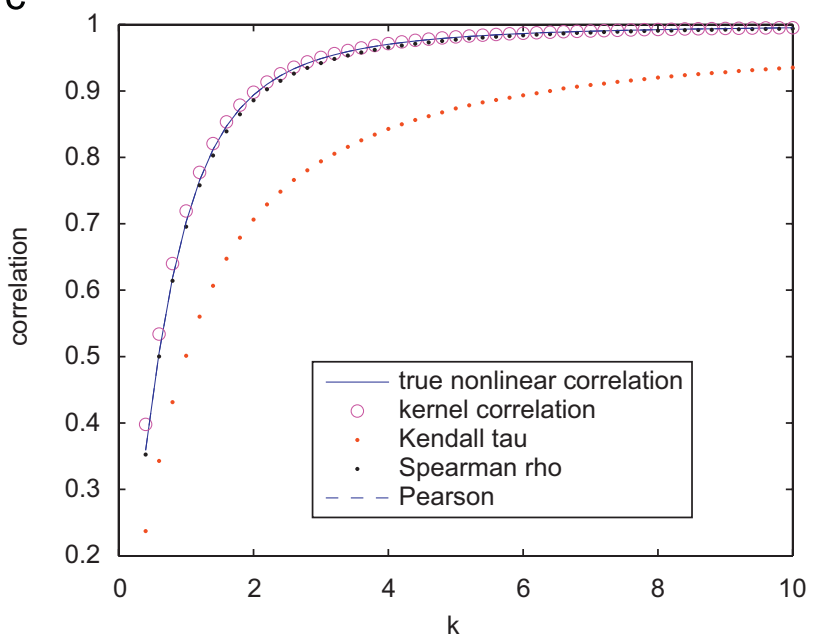

Fig. 1. (a) Example 1, Model I: $X^{(2)}=\cos \left(\pi X^{(1)}\right)+\varepsilon / k$. (b) Example 1, Model II: $X^{(2)}=\exp \left(-\left|X^{(1)}\right|\right)+\varepsilon / k$. (c) Example 1 , Model III: $X^{(2)}=X^{(1)}+\varepsilon / k$. 
Table 1

Correlation coefficients for the first and the second pairs of linear canonical variates and kernel canonical variates for Example 2

\begin{tabular}{lll}
\hline Average correlation coefficient & LCCA (s.e.) & KCCA (s.e.) \\
\hline Between 1st pair canonical variates & $0.0614(0.0046)$ & $0.9978(0.0000)$ \\
Between 2nd pair canonical variates & $0.0175(0.0031)$ & $0.9927(0.0001)$
\end{tabular}

a

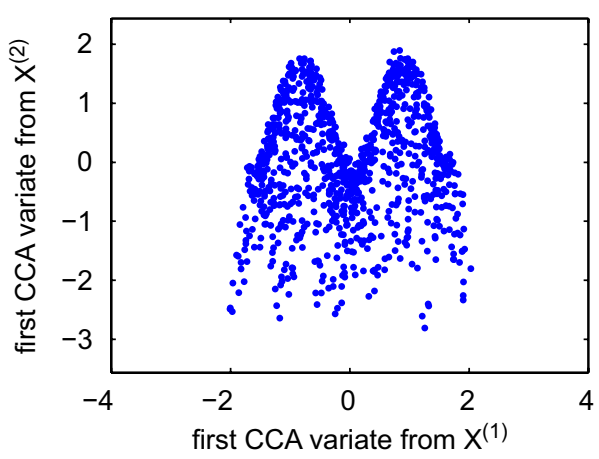

C

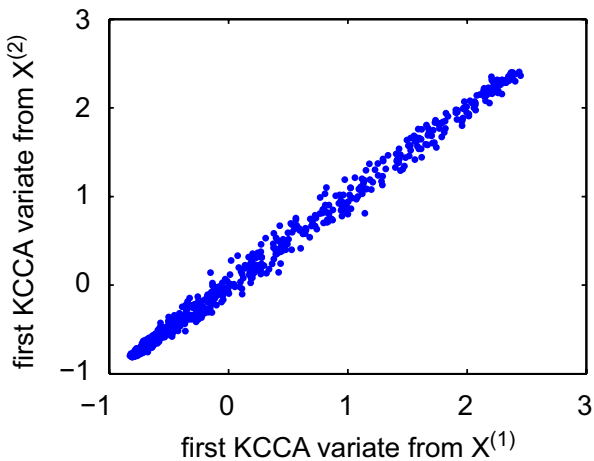

b

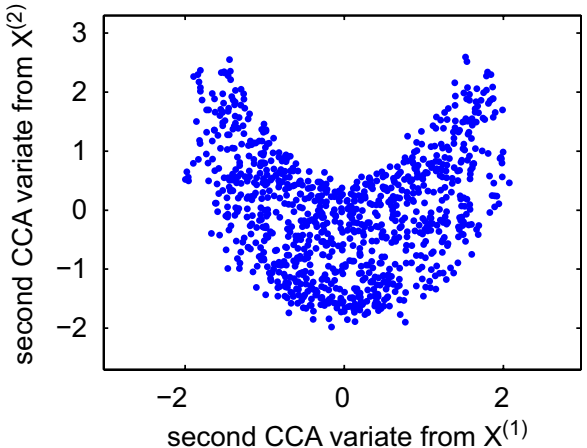

d

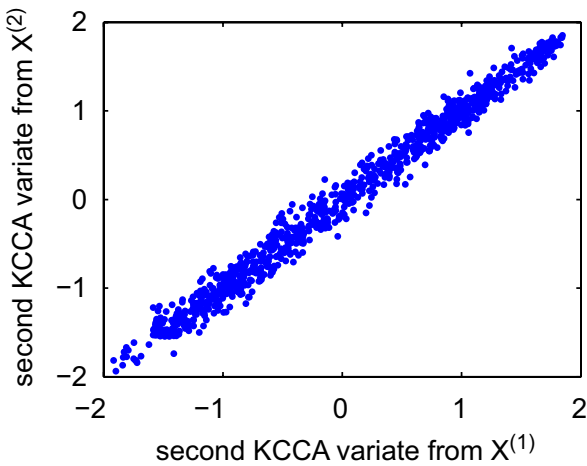

Fig. 2. Scatter plots of the first and second pairs of linear canonical variates ((a) and (b)) and kernel canonical variates ((c) and (d)) for Example 2.

are, respectively, data scatters of the first and the second pair of kernel canonical variates. They show strong linear correlation between KCCA-found variates of the two sets of variables. With the kernel transformation, the distribution of pairs of canonical variates is indeed prominently depicted as elliptically symmetrically distributed. This phenomenon hints that many multivariate data analysis tools, which originally are constrained by Gaussian or elliptical symmetric distribution assumption, now can extend to work on kernel data. Also the strong linear relationship shown in Fig. 2(c) and (d) suggests that linear learning algorithms can be applied to KCCA-found variates for various learning tasks. Below we give an example of applying the classical Fisher linear discriminant analysis on top of KCCA-found variates for classification.

Example 3 (Nonlinear dimension reduction and discriminant analysis). In this example, kernel canonical variates are employed as discriminant variates for multiclass classification. Data set used is the pen-based recognition ofhand-written digits (Asuncion and Newman, 2007). There are 10 classes in this data set. We first construct a 10 -dimensional class indicator variable " $x^{(2)}=$ $\left(c_{1}, \ldots, c_{10}\right)$ " as follows:

$$
c_{i}= \begin{cases}1 & \text { if an instance input is from the } i \text { th class, i.e., from digit } i-1, \\ 0 & \text { otherwise. }\end{cases}
$$

The number of training and testing instances are 7494 and 3498, respectively. For each instance there are 16 input measurements (i.e., $x^{(1)}$ is 16 -dimensional) and a corresponding class label from $\{0,1,2, \ldots, 9\}$. A Gaussian kernel with the rule-of-thumb window width suggested in Section 3 is used, and a random subset from training inputs of size 750 (stratified over 10 classes with 75 each) is adopted as kernel basis, i.e., the training and testing kernel data matrices have the sizes $7494 \times 750$ and $3498 \times 750$, respectively. We keep the class label variable $x^{(2)}$ as in (12) without kernel transformation. Then, the LCCA procedure is acting on the reduced kernel training inputs and their associated class labels. For a 10-class problem, it leads to nine canonical variates as discriminant variates. The KCCA heuristic for discriminant analysis goes as follows. It first extends the data to nonlinear high 

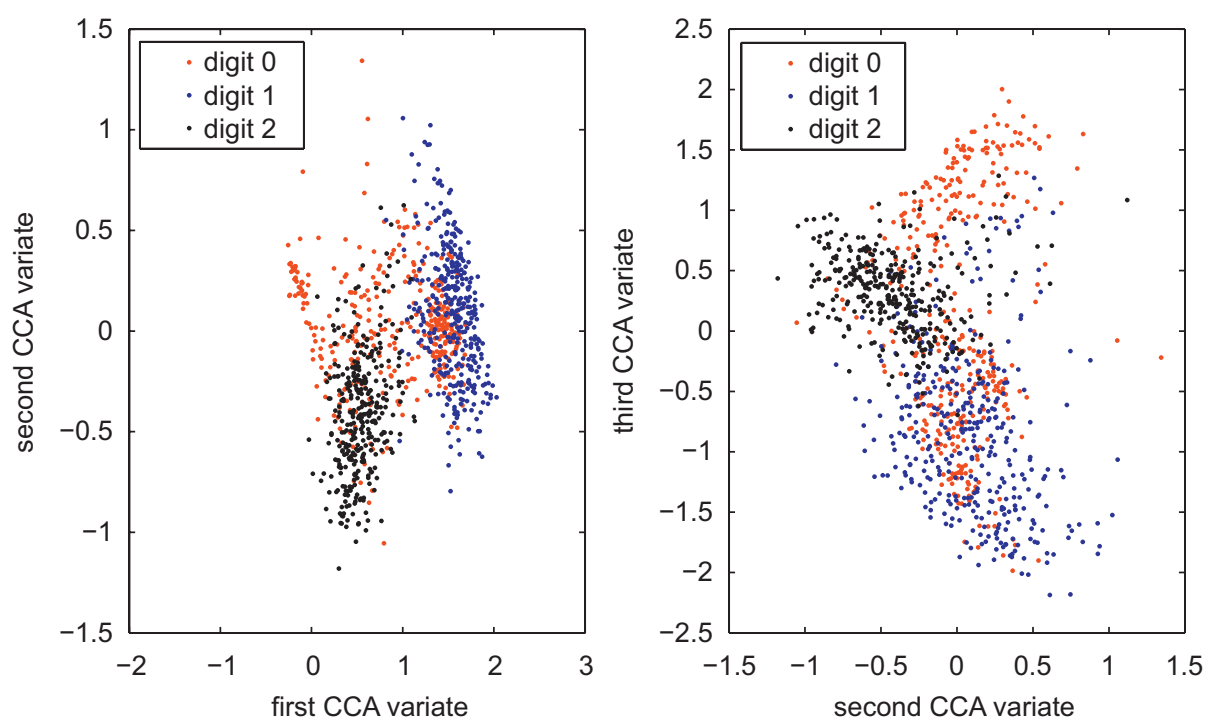

Fig. 3. Scatter plots of leading linear canonical variates with Pendigits data.
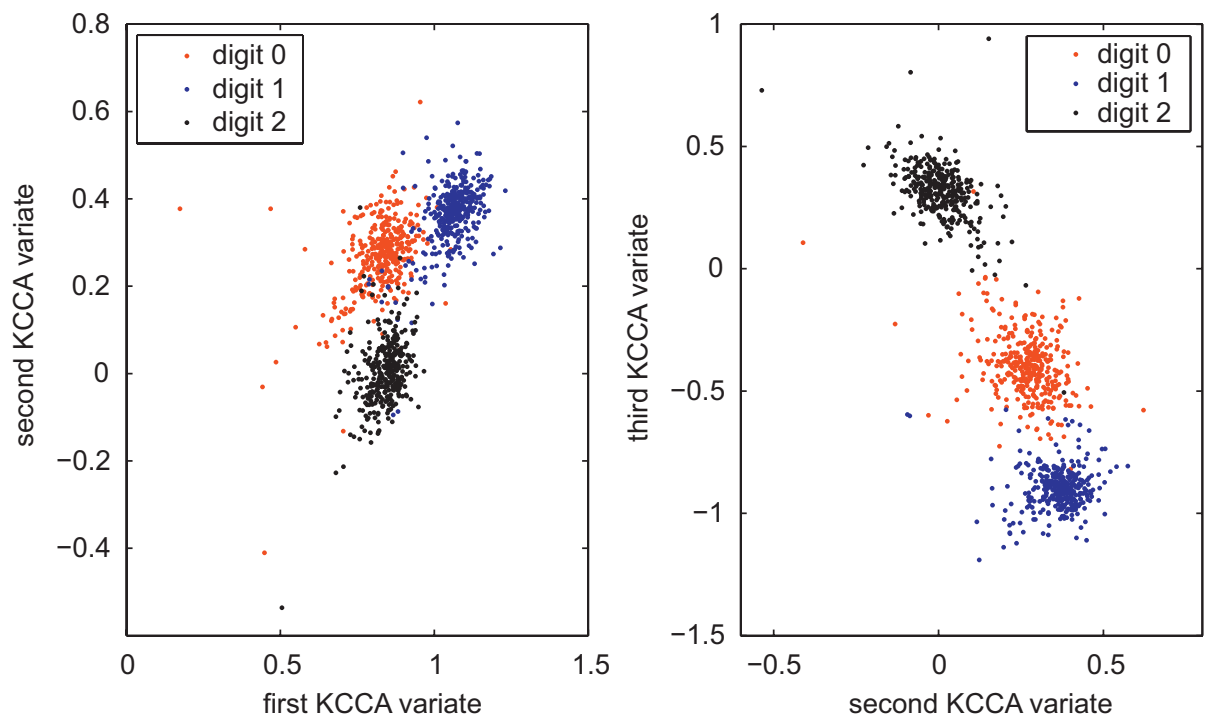

Fig. 4. Scatter plots of leading kernel canonical variates with Pendigits data.

inputs by augmenting the original input measurements in $\mathfrak{R}^{16}$ through high input measurements in $\mathfrak{R}^{750}$ using 750 random kernel basis functions. Then it cuts down the dimensionality from 750 to nine kernel canonical variates by canonical correlation analysis for discriminant purpose. Scatter plots of test instances projected along the linear canonical directions and along kernel canonical directions are given in Figs. 3 and 4, respectively. To avoid excessive ink we only display test instances from digits 0 to 2. Different classes are labeled with distinct colors. It can be seen that the scatters of kernel canonical variates are better elliptically distributed than the linear canonical variates, and also that the kernel canonical variates have better between-class dispersion with much less class overlaps. The classification is made by Fisher's linear discriminant analysis on top of the KCCAfound variates, which have the dimensionality 9 for a 10 -class problem. A test instance is assigned to the class with the closest class center (according to Mahalanobis distance). The accuracy of the kernel classification on test data can achieve the rate $98.09 \%$ (with standard error $0.06 \%$ over 10 replicate samplings of random bases in the regularization step).

\subsection{Test of independence between two sets of variables}

In this section we generalize the use of Bartlett's test of independence (Bartlett, 1947a,b) to kernel data.

$$
\left(n-\frac{1}{2}\left(m_{1}+m_{2}+1\right)\right) r_{\log }^{\kappa} \sim \chi_{\left(m_{1}-1\right)\left(m_{2}-1\right)}^{2},
$$


Table 2

Power estimates (or type-I error estimate in III-1 case) for testing independence of $(X, Y)$ for several distributions based on 100 replicate runs

\begin{tabular}{|c|c|c|c|c|c|}
\hline Case & $n$ & LCCA (s.e.) & KCCA (s.e.) & D\&N spline 2 & D\&N spline 3 \\
\hline I & $500^{*}$ & $0.42(0.049)$ & $1.00(0.000)$ & 1.00 & 1.00 \\
\hline II & 500 & $0.02(0.014)$ & $1.00(0.000)$ & 0.99 & 0.99 \\
\hline III- $1, \rho=0$ & 500 & $0.06(0.024)$ & $0.04(0.020)$ & NA & NA \\
\hline III- $2, \rho=0.2$ & 500 & $0.99(0.010)$ & $0.96(0.020)$ & NA & NA \\
\hline III- $3, \rho=0.5$ & 500 & $1.00(0.000)$ & $1.00(0.000)$ & NA & NA \\
\hline III- $4, \rho=0.8$ & 500 & $1.00(0.000)$ & $1.00(0.000)$ & 0.99 & 0.73 \\
\hline IV & 500 & $0.62(0.049)$ & $1.00(0.000)$ & 1.00 & 1.00 \\
\hline V & 500 & $0.37(0.048)$ & $1.00(0.000)$ & 1.00 & 1.00 \\
\hline VI & 500 & $0.09(0.029)$ & $1.00(0.000)$ & NA & NA \\
\hline
\end{tabular}

The significance level is $\alpha=0.05$ The D\&N columns are power estimates taken from Dauxois and Nkiet (1998) using orders 2 and 3 splines. (For case I, the D\&N has used sample sizes 200 and 400, instead of 500. "NA" indicates that they did not have the experiment.)

where $r_{\log }^{\kappa}=-\sum_{v} \log \left(1-\left(\hat{\rho}_{v}^{\kappa}\right)^{2}\right)$ with $\hat{\rho}_{v}^{\kappa}$ s seing the sample estimates of kernel canonical correlation coefficients, and $m_{1}$ and $m_{2}$ are the numbers of kernel bases for the first and the second set of variables, respectively. Technical details to justify (13) are placed in Appendix A.

Remark 1. There are two major differences in our independence test from Dauxois and Nkiet's. One is the baseline association measure used. Based on the association measure (10), they have used the following test statistic for independence:

$$
\frac{n \sum_{v}\left(\hat{\rho}_{v}^{\mathrm{spl}}\right)^{2}}{1+\sum_{v}\left(\hat{\rho}_{v}^{\mathrm{spl}}\right)^{2}} \sim \chi_{\left(m_{1}-1\right)\left(m_{2}-1\right)}^{2},
$$

where $\hat{\rho}_{v}^{\text {spl, }}$ s are the sample estimates of nonlinear canonical correlation coefficients using splines, and $m_{1}$ and $m_{2}$ are the numbers of spline bases for the first and the second set of variables, respectively. Instead of (14) we use a logarithm-based test statistic (13), which is the maximum log-likelihood ratio of the null versus the alternative under Gaussian distribution assumption. Though we do not need any Gaussian assumption for the asymptotic $\chi^{2}$ distribution for the independence test (13) (see Appendix A), the heuristic behind our test statistic is that low-dimensional projections from high-dimension data are approximately Gaussian (Huang and Hwand, 2006). The other difference is the systems of basis sets used for nonlinear canonical analysis. Dauxois and Nkiet have used splines, and here we use kernel functions. The difference in basis sets leads to different implementing procedures and different computing load. The kernel algorithm in this article does not require much of extra programming needs, we use the classical LCCA steps on kernel data.

In Example 4, tests of two sets of variables for several distributions are carried out using KCCA. The kernel data regularization is done by SVD-based optimal basis extracting $99 \%$ of data variation. The first five distributions in this study are taken from Dauxois and Nkiet (1998) and the last one is similar to Example 2 in Section 4.1 but without the additive noise.

Example 4 (Independence tests). In the first five cases, we are interested in testing the independence between $X$ and $Y$ that are both one-dimensional. In the last case, VI, the independence between two vectors $X$ and $Y$ are tested, where both $X$ and $Y$ are two-dimensional:

I. $X \sim \mathrm{N}(0,1)$ and $Y=X^{2}$;

II. $(X, Y) \sim$ uniform distribution on the unit disk;

III. $(X, Y) \sim$ bivariate standard normal with correlation $\rho$;

IV. $(X, Y) \sim$ a mixture distribution: $P_{\theta}=\theta Q_{1}+(1-\theta) Q_{2}$ with $\theta=0.5$, where $Q_{1}$ is the distribution of $\left(X, X^{2}\right)$ with $X$ a univariate standard normal and $Q_{2}$ is the bivariate standard normal with correlation $\rho=0.25$;

V. a mixture distribution: $P_{\theta}=\theta Q_{1}+(1-\theta) Q_{2}$ with $\theta=0.75$ and

VI. $X=\left(X_{1}, X_{2}\right)$ has a bivariate uniform distribution over $(-2,2)^{2}$ and $Y=\left(X_{1}^{2}, \cos \left(\pi X_{2}\right)\right)$.

Five hundreds sample points are drawn from each of the above distributions. The total number of replicate runs is 100 . The power estimates (or type-I error estimate for case III-1, where $\rho=0$ ) and their standard errors for the independence test are reported in Table 2. Results in the LCCA column are from Barlett's independence test based on linear canonical correlation coefficients, results in the KCCA column are also from Barlett's independence test but based on kernel canonical correlation coefficients, and results in the D\&N columns are taken from Dauxois and Nkiet (1998) with order 2 (linear) and order 3 (quadratic) splines. It can be seen that the KCCA-based test has power of 1 in all cases except for case III- 2 with $\rho=0.2$; while the LCCA catches the relation only when the linearity is present (cases III-2-4). For the type I error (case III-1, $\rho=0$ ), the KCCA-based test has a bit underestimated the type-I error, while the LCCA-based test has a bit overestimated the type-I error, but the difference is not prominent. The KCCA performs about the same as the orders 2 and 3 spline approximations in nonlinear association models, 
but much better than the LCCA-based tests. In linear association models, the nonlinear and nonparametric KCCA still does well in all the experimental cases, while the order 3 spline has a much lower power in the linear case with $\rho=0.8$ and has no reports for other linear cases with smaller $\rho$ 's. In general, the KCCA provides an effective alternative choice for nonparametric independence test. It performs well for linear and nonlinear association models and the test is quite easy to compute.

\section{Conclusion}

In this paper, we discuss the use of kernel method for studying relation of two sets of variables via nonlinear canonical analysis. The key contribution is twofold. One is to place the KCCA working procedure in an LCCA framework so that the implementation efforts aim to be minimized. The other is the KCCA applications including a new proposal of independence test and a study of various association measures to show their nonparametric nature as well as nonparametric ability to allow for hybrid with linear learning algorithms.

\section{Acknowledgement}

The authors thank Hwang, Chii-Ruey for helpful discussion.

\section{Appendix A. Appendix}

In this appendix we give some basic theory of KCCA to justify (I) its working procedure by carrying out the classical LCCA on kernel data and (II) its usage for independence test given in (13). For simplicity we assume that $\mathscr{X}$ is compact. Let $X=\left(X^{(1)}, X^{(2)}\right)$ be a random vector having the distribution $P$, and let $P_{1}$ and $P_{2}$ be the marginal distributions for $X^{(1)}$ and $X^{(2)}$, respectively. We make the following assumptions concerning the underlying distribution:

(D1) Assume the probability space $(\mathscr{X}, \mathscr{B}, P)$ considered is such that the Hilbert space $L_{2}(\mathscr{X}, \mathscr{B}, P)$ (also denoted by $L_{2}(P)$ for short) is separable. ${ }^{1}$

(D2) Assume that $P, P_{1}$ and $P_{2}$ have continuous probability density functions with respect to the Lebesgue measure and satisfy $\int_{\mathscr{X}_{i}} p_{i}^{2}\left(x^{(i)}\right) \mathrm{d} x^{(i)}<\infty$. This finiteness is to prevent the underlying density functions from going to infinity too fast near the boundaries of $\mathscr{X}_{i}$.

(D3) Assume that the support of $P_{i}$ covers the entire $\mathscr{X}_{i}$ for $i=1,2$. If not, restrict $\mathscr{X}_{i}$ to the support of $P_{i}$.

The KCCA can be formulated as a special case of a more general NCCA. The theoretical foundation of NCCA of two sets of random variables can be derived through the canonical analysis of two separable Hilbert subspaces $L_{2}\left(P_{1}\right)$ and $L_{2}\left(P_{2}\right)($ Dauxois and Nkiet, 1998). The canonical analysis of two separable Hilbert subspaces is to look for an orthonormal system for each subspace so that the correlations (in terms of inner product) between the two systems are sequentially maximized, or equivalently the canonical angles between the two orthonormal systems are sequentially minimized. This is analogous to the classical LCCA of two Euclidean subspaces.

In this appendix we will put the KCCA into the general framework of canonical analysis of two reproducing kernel Hilbert subspaces to validate its working procedure and asymptotic distribution.

\section{A.1. KCCA working procedure}

Assume that the kernels $\kappa_{1}$ and $\kappa_{2}$, defined on $\mathscr{X}_{1} \times \mathscr{X}_{1}$ and $\mathscr{X}_{2} \times \mathscr{X}_{2}$, respectively, have the following spectrum decompositions:

$$
\kappa_{1}\left(x^{(1)}, u^{(1)}\right)=\sum_{v} \lambda_{v}^{(1)} \phi_{v}\left(x^{(1)}\right) \phi_{v}\left(u^{(1)}\right) \quad \text { and } \quad \kappa_{2}\left(x^{(2)}, u^{(2)}\right)=\sum_{v} \lambda_{v}^{(2)} \psi_{v}\left(x^{(2)}\right) \psi_{v}\left(u^{(2)}\right)
$$

where $\lambda_{1}^{(i)} \geqslant \lambda_{2}^{(i)} \geqslant \cdots \geqslant 0, \sum_{v} \lambda_{v}^{(i)}<\infty$ for $i=1,2$, and $\phi$ 's and $\psi$ 's are orthonormal systems (with respect to the Lebesgue measure) on $\mathscr{X}_{1}$ and $\mathscr{X}_{2}$, respectively. A common approach for KCCA (e.g., Gretton et al., 2003; Hardoon et al., 2004; Kuss and Graepel, 2003; Gretton et al., 2005) is to map the original input space to a high dimensional feature space $\mathscr{Z}{ }_{i}, i=1,2$, via the feature maps

$$
\Phi: \mathscr{X}_{1} \mapsto \mathscr{Z}_{1} \text { and } \Psi: \mathscr{X}_{2} \mapsto \mathscr{Z}_{2}
$$

where

$$
\begin{aligned}
z^{(1)} & :=\Phi\left(x^{(1)}\right):=\left(\sqrt{\lambda_{1}^{(1)}} \phi_{1}\left(x^{(1)}\right), \sqrt{\lambda_{2}^{(1)}} \phi_{2}\left(x^{(1)}\right), \ldots\right) \text { and } \\
z^{(2)} & :=\Psi\left(x^{(1)}\right):=\left(\sqrt{\lambda_{1}^{(2)}} \psi_{1}\left(x^{(2)}\right), \sqrt{\lambda_{2}^{(2)}} \psi_{2}\left(x^{(2)}\right), \ldots\right) .
\end{aligned}
$$

${ }^{1}$ A separable Hilbert space has a countable orthonormal basis and elements therein can be expanded in terms of this countable basis. 
Instead of the spectrum-based feature spaces $\mathscr{Z}_{1}$ and $\mathscr{Z}_{2}$, we will introduce their isometric isomorphisms. For a given positive definite kernel $\kappa_{1}$, its associated Hilbert space, denoted by $\mathscr{H}_{\kappa_{1}}$, consists of all finite kernel mixtures, $\sum_{j=1}^{m} a_{j} \kappa_{1}\left(x^{(1)}, u_{j}^{(1)}\right)$, and

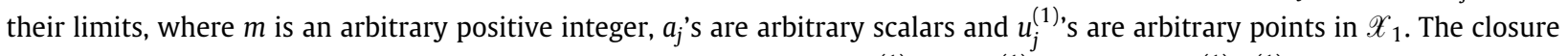
is taken with respect to the norm induced by the inner product: $\left\langle\kappa_{1}\left(x^{(1)}, \cdot\right), \kappa_{1}\left(u^{(1)}, \cdot\right)\right\rangle_{\mathscr{H}_{\kappa_{1}}}=\kappa_{1}\left(x^{(1)}, u^{(1)}\right)$. This Hilbert space is known as an RKHS. Similarly, the associated space $\mathscr{H}_{\kappa_{2}}$ consists of all finite kernel mixtures by $\kappa_{2}$ and their limits. Consider an alternative feature map $\Gamma_{i}: \mathscr{X}_{i} \mapsto \mathscr{H}_{\kappa_{i}}$ given by

$$
\chi^{(i)} \mapsto \Gamma_{i}\left(x^{(i)}\right):=\kappa_{i}\left(x^{(i)}, \cdot\right)
$$

The original input space $\mathscr{X}_{1} \oplus \mathscr{X}_{2}$ is then embedded into a new feature space $\mathscr{H}_{\kappa_{1}} \oplus \mathscr{H}_{\kappa_{2}}$ via the transformation $\Gamma=\Gamma_{1} \oplus \Gamma_{2}$. Each input point $x^{(i)} \in \mathscr{X}_{i}$ is mapped to an element $\kappa_{i}\left(x^{(i)}, \cdot\right) \in \mathscr{H}_{\kappa_{i}}$. Let

$$
\mathscr{J}_{i}: \mathscr{Z}_{i} \mapsto \mathscr{H}_{\kappa_{i}}
$$

be a map from the spectrum-based feature space $\mathscr{Z}_{i}$ to the kernel associated Hilbert space $\mathscr{H}_{\kappa_{i}}$ defined by $\mathscr{J}_{1}\left(\Phi\left(x^{(1)}\right)\right)=\kappa_{1}\left(x^{(1)}, \cdot\right)$ and $\mathscr{J}_{2}\left(\Psi\left(x^{(2)}\right)\right)=\kappa_{2}\left(x^{(2)}, \cdot\right)$. By condition (K3) below and the reproducing property

$$
\left\langle\kappa_{i}\left(x^{(i)}, \cdot\right), f(\cdot)\right\rangle_{\mathscr{H}_{\kappa_{i}}}=f\left(x^{(i)}\right), \quad \forall f \in \mathscr{H}_{\kappa_{i}} \text { and } \forall x^{(i)} \in \mathscr{X}_{i},
$$

it is easy to verify that $\mathscr{J}_{i}$ is a one-to-one linear transformation satisfying

$$
\left\|z^{(i)}\right\|_{\mathscr{Z}_{i}}^{2}=\kappa_{i}\left(x^{(i)}, x^{(i)}\right)=\left\|\kappa_{i}\left(x^{(i)}, \cdot\right)\right\|_{\mathscr{H}_{\kappa_{i}}}^{2}=\left\|\mathscr{J}_{i}\left(z^{(i)}\right)\right\|_{\mathscr{H}_{\kappa_{i}}}^{2} .
$$

Thus, the two feature representations (15) and (16) are equivalent in the sense of isometric isomorphism. For theoretical development we will work in the framework of the feature spaces $\mathscr{H}_{\kappa_{i}}$ instead of $\mathscr{Z}_{i}$. We assume that the reproducing kernels employed are:

(K1) symmetric in the sense of $\kappa_{i}\left(x^{(i)}, u^{(i)}\right)=\kappa_{i}\left(u^{(i)}, x^{(i)}\right)$ and Lebesgue-measurable;

(K2) of trace type, i.e., the corresponding $\lambda$ 's satisfy $\sum_{v} \lambda_{v}^{(i)}<\infty$;

(K3) for $x^{(i)} \neq u^{(i)}, \kappa_{i}\left(x^{(i)}, \cdot\right) \neq \kappa_{i}\left(u^{(i)}, \cdot\right), i=1,2$ and

(K4) of translation type, i.e., $\kappa_{i}\left(x^{(i)}, u^{(i)}\right)=\kappa_{i}\left(x^{(i)}-u^{(i)}\right)$, and having tail decay to zero as $\left\|x^{(i)}-u^{(i)}\right\| \rightarrow 0$.

For given window widths $\sigma_{1}$ and $\sigma_{2}$, let

$$
\begin{aligned}
& V_{\kappa_{1}}^{c}=\left\{f \in \mathscr{H}_{\kappa_{1}}: f\left(X^{(1)}\right) \in L_{2}\left(P_{1}\right) \text { and } E\left(f\left(X^{(1)}\right)\right)=0\right\} \quad \text { and } \\
& V_{\kappa_{2}}^{c}=\left\{g \in \mathscr{H}_{\kappa_{2}}: g\left(X^{(2)}\right) \in L_{2}\left(P_{1}\right) \text { and } E\left(g\left(X^{(2)}\right)\right)=0\right\}
\end{aligned}
$$

be the collection of centered $L_{2}$ variates of $f\left(X^{(1)}\right)$ with $f \in \mathscr{H}_{\kappa_{1}}$ and $g\left(X^{(2)}\right)$ with $g \in \mathscr{H}_{\kappa_{2}}$, respectively. Let $V_{\kappa_{1}}$ and $V_{\kappa_{2}}$ be all noncentered $L_{2}$ variates in $\mathscr{H}_{\kappa_{1}}$ and $\mathscr{H}_{\kappa_{2}}$, respectively. The KCCA is the study of canonical analysis between the two central subspaces $V_{\kappa_{1}}^{c}$ and $V_{\kappa_{2}}^{c}$ placed in the space $V_{\kappa_{1} \oplus \kappa_{2}}$, where

$$
\left(\kappa_{1} \oplus \kappa_{2}\right)(x, u)=\kappa_{1}\left(x^{(1)}, u^{(2)}\right)+\kappa_{2}\left(x^{(2)}, u^{(2)}\right) .
$$

The theory of RKHS is a powerful tool in statistics and probability, and particularly in stochastic processes, nonparametric inference and the recent burst of kernel learning algorithms. See Berlinet and Thomas-Agnan (2004) for a clear and up-to-date account of theory, examples and computational aspects of RKHS. Below we will formulate and analyze the nonlinear relation between $X^{(1)}$ and $X^{(2)}$ through the canonical analysis of the two central subspaces $V_{\kappa_{1}}^{c}$ and $V_{\kappa_{2}}^{c}$. Let $L_{2}^{c}\left(P_{1}\right)=\left\{f \in L_{2}\left(P_{1}\right): E(f)=0\right\}$ and $L_{2}^{c}\left(P_{2}\right)=\left\{g \in L_{2}\left(P_{2}\right): E(g)=0\right\}$, which are subspaces consisting of all zero mean $L_{2}$ variates. In the LCCA we are seeking leading pairs of linear canonical variates $\left\{\alpha_{i}^{\prime}\left(X^{(1)}-E X^{(1)}\right) \in L_{2}^{c}\left(P_{1}\right), \beta_{i}^{\prime}\left(X^{(2)}-E X^{(2)}\right) \in L_{2}^{c}\left(P_{2}\right)\right\}_{i=1}^{\ell}$, which are orthonormal in the metrics $L_{2}\left(P_{1}\right)$ and $L_{2}\left(P_{2}\right)$, respectively, ${ }^{2}$ and which sequentially maximize the correlations between $\alpha^{\prime} X^{(1)}$ and $\beta^{\prime} X^{(2)}$. Similarly for its nonlinear kernel generalization, we are seeking leading pairs of canonical variates $\left\{f_{i}\left(X^{(1)}\right) \in V_{\kappa_{1}}^{c}, g_{i}\left(X^{(2)}\right) \in V_{\kappa_{2}}^{c}\right\}_{i=1}^{\ell}$, which are orthonormal in the metrics $L_{2}\left(P_{1}\right)$ and $L_{2}\left(P_{2}\right)$, respectively, and which sequentially maximize the correlations between $f\left(X^{(1)}\right)$ and $g\left(X^{(2)}\right)$. That is, we are seeking pairs $f_{i} \in V_{\kappa_{1}}^{c}$ and $g_{i} \in V_{\kappa_{2}}^{c}$ to sequentially maximize $\operatorname{cov}\left(f_{i}, g_{i}\right) \operatorname{subject}$ to $\operatorname{cov}\left(f_{i}, f_{i^{\prime}}\right)=\delta_{i i^{\prime}}$ and $\operatorname{cov}\left(g_{i}, g_{i^{\prime}}\right)=\delta_{i i^{\prime}}$. The corresponding coefficients are called kernel canonical correlation coefficients and denoted by $\rho_{i}^{\kappa}=\operatorname{cor}\left(f_{i}, g_{i}\right)$ in descending order, i.e., $\rho_{1}^{\kappa} \geqslant \rho_{2}^{\kappa} \geqslant \rho_{3}^{\kappa} \geqslant \cdots \geqslant 0$. The KCCA then consists of a triple:

$$
\left\{\left(\rho_{v}^{\kappa}, f_{v}\left(X^{(1)}\right), g_{v}\left(X^{(2)}\right)\right), v=0,1,2, \ldots\right\},
$$

\footnotetext{
${ }^{2}$ For $\left\{f_{1}, \ldots, f_{\ell}\right\}$ being orthonormal with respect to the metric $L_{2}\left(P_{1}\right)$, it means that $E_{P_{1}}\left(f_{i} f_{i^{\prime}}\right):=\int_{\mathscr{X}_{1}} f_{i}\left(x^{(1)}\right) f_{i^{\prime}}\left(x^{(1)}\right) \mathrm{d} P_{1}\left(x^{(1)}\right)=\delta_{i i^{\prime}}$, and same for being orthonormal with respect to the metric $L_{2}\left(P_{2}\right)$.
} 
where $f_{v}$ and $g_{v}$ are $L_{2}$-variates from $\mathscr{H}_{\kappa_{1}}$ and $\mathscr{H}_{\kappa_{2}}$, respectively. The trivial canonical terms $\rho_{0}^{\kappa}, f_{0}=1$ and $g_{0}=1$ are added for completeness reason and can be easily avoided by restricting the KCCA to centered variates. To put the KCCA in practice and for implementation by a computer, one has to work on a discretization of KCCA by a pair of finite systems in $\mathscr{H}_{\kappa_{1}}$ and $\mathscr{H}_{\kappa_{2}}$. Such a pair of systems have to become essentially dense in $L_{2}\left(P_{1}\right)$ and $L_{2}\left(P_{2}\right)$, as $\sigma_{1}, \sigma_{2} \downarrow 0$ with $n \rightarrow \infty$. For $\ell \in \mathbb{N}$, consider the following systems (as functions of $x^{(1)}$ and $x^{(2)}$ )

$$
\begin{aligned}
& \kappa_{1}\left(x^{(1)}, u_{v}^{(1)}\right), \quad u_{v}^{(1)} \in \mathscr{X}_{1}, \quad v=1, \ldots, p_{\ell}, \\
& \kappa_{2}\left(x^{(2)}, u_{\mu}^{(2)}\right), \quad u_{\mu}^{(2)} \in \mathscr{X}_{2}, \quad \mu=1, \ldots, q_{\ell},
\end{aligned}
$$

where $\left\{u_{v}^{(1)}\right\}_{v=1}^{p_{\ell}}$ and $\left\{u_{\mu}^{(2)}\right\}_{\mu=1}^{q_{\ell}}$ are sequences of distinct points essentially dense in $\mathscr{X}_{1}$ and $\mathscr{X}_{2}$, respectively, as $p_{\ell}, q_{\ell} \rightarrow \infty$.

Proposition 1. Assume $\kappa_{1}$ and $\kappa_{2}$ satisfy conditions (K1)-(K4) and the sequences of points $\left\{u_{v}^{(1)}\right\}_{v=1}^{p_{\ell}}$ and $\left\{u_{\mu}^{(2)}\right\}_{\mu=1}^{q_{\ell}}$ are as stated above. Then, the two systems

$$
\left\{\kappa_{1}\left(X^{(1)}, u_{v}^{(1)}\right)\right\}_{1 \leqslant v \leqslant p_{\ell}} \text { and }\left\{\kappa_{2}\left(X^{(2)}, u_{\mu}^{(2)}\right)\right\}_{1 \leqslant \mu \leqslant p_{\ell}}
$$

are linear independent in $L_{2}\left(P_{1}\right)$ and $L_{2}\left(P_{2}\right)$, respectively. Furthermore, assume that $\sigma_{1}, \sigma_{2} \rightarrow 0$ and $p_{\ell}, q_{\ell} \rightarrow \infty$ as $n \rightarrow \infty$, then the systems are essentially dense in $L_{2}\left(P_{1}\right)$ and $L_{2}\left(P_{2}\right)$, respectively.

Proof. Condition (K3) implies that $\left\{\kappa_{1}\left(X^{(1)}, u_{v}^{(1)}\right)\right\}_{1 \leqslant v \leqslant p_{\ell}}$ and $\left\{\kappa_{2}\left(X^{(2)}, u_{\mu}^{(2)}\right)\right\}_{1 \leqslant \mu \leqslant q_{\ell}}$ are linear independent systems in $L_{2}\left(P_{1}\right)$ and $L_{2}\left(P_{2}\right)$, respectively. Next we have to show that the systems are essentially dense in $L_{2}\left(P_{1}\right)$ and $L_{2}\left(P_{2}\right)$. For fixed $\sigma_{1}$ and $\sigma_{2}$, any $f \in V_{\kappa_{1}}$ and an arbitrary $\varepsilon>0$, there exist a positive integer $m$, a sequence of points $\left\{x_{i}^{(1)}\right\}_{i=1}^{m}$ and a sequence of numbers $\left\{a_{i}\right\}_{i=1}^{m}$ such that $\left\|f(\cdot)-\sum_{i=1}^{m} a_{i} \kappa_{1}\left(\cdot, x_{i}^{(1)}\right)\right\|_{\mathscr{H}_{\kappa_{1}}}<\varepsilon$. As points $\left\{u_{v}^{(1)}\right\}_{v=1}^{p_{\ell}}$ are essentially dense in $\mathscr{X}_{1}$, we can find for each $x_{i}^{(1)}$, with a fixed but large enough $\ell$, a point from the set $\left\{u_{v}^{(1)}\right\}_{v=1}^{p_{\ell}}$, denoted by $u_{i, \ell}^{(1)}$ that $\left\|\kappa_{1}\left(\cdot, x_{i}^{(1)}\right)-\kappa_{1}\left(\cdot, u_{i, \ell}^{(1)}\right)\right\|_{\mathscr{H}_{\kappa_{1}}}<\varepsilon /\left(m\left|a_{i}\right|\right)$. Then, we have

$$
\left\|f(\cdot)-\sum_{i=1}^{m} a_{i} \kappa_{1}\left(\cdot, u_{i, \ell}^{(1)}\right)\right\|_{\mathscr{H}_{\kappa_{1}}}<2 \varepsilon .
$$

Thus, the system $\left\{\kappa_{1}\left(X^{(1)}, u_{v}^{(1)}\right)\right\}_{1 \leqslant v \leqslant p_{\ell}}$ is essentially dense in $V_{\kappa_{1}}$. Similarly, one can show that the system $\left\{\kappa_{2}\left(X^{(2)}, u_{v}^{(2)}\right)\right\}_{1 \leqslant v \leqslant p_{\ell}}$ is essentially dense in $V_{\kappa_{2}}$. It is also known that as $\sigma_{1}, \sigma_{2} \downarrow 0, V_{\kappa_{1}}$ and $V_{\kappa_{2}}$ are essentially dense in $L_{2}\left(P_{1}\right)$ and $L_{2}\left(P_{2}\right)$, respectively.

As the marginal distributions $P_{i}$ are assumed to have continuous pdf and to have $\operatorname{support}\left(P_{i}\right)=\mathscr{X}_{i}$, we have the following lemma.

Proposition 2. Let $X_{j}^{(i)}, j=1,2, \ldots$, be i.i.d. from a continuous distribution $P_{i}, i=1,2$, satisfying conditions (D1)-(D3). Then the collection of points $\left\{X_{j}^{(i)}\right\}_{j=1}^{\infty}$ are distinct and dense in $\mathscr{X}_{i}$ for $i=1,2$ almost surely.

Let $\left\{x_{1}^{(1)}, \ldots, x_{p_{\ell}}^{(1)}\right\}$ and $\left\{x_{1}^{(2)}, \ldots, x_{q_{\ell}}^{(2)}\right\}$ be some realizations of $X^{(1)}$ and $X^{(2)}$, respectively. With the properties given in Propositions 1 and 2 , the following systems:

$$
\begin{aligned}
& F^{\ell}\left(X^{(1)}\right):=\left(\kappa_{1}\left(X^{(1)}, x_{1}^{(1)}\right), \ldots, \kappa_{1}\left(X^{(1)}, x_{p_{\ell}}^{(1)}\right)\right), \\
& G^{\ell}\left(X^{(2)}\right):=\left(\kappa_{2}\left(X^{(2)}, x_{1}^{(2)}\right), \ldots, \kappa_{2}\left(X^{(2)}, x_{q_{\ell}}^{(2)}\right)\right)
\end{aligned}
$$

are essentially dense in $L_{2}\left(P_{1}\right)$ and $L_{2}\left(P_{2}\right)$, respectively. By the discussion in Dauxois and Nkiet (1998, Section 4.1), the KCCA of $X^{(1)}$ and $X^{(2)}$ can be approximated (in terms of uniform convergence) by the sequence of LCCAs of $F^{\ell}\left(X^{(1)}\right)$ and $G^{\ell}\left(X^{(2)}\right)$. Note that such an underlying sequence of LCCA approximations depends on the choice of linear independent systems given in (19) and (20). With the choice $\left\{x_{1}^{(1)}, \ldots, x_{p_{\ell}}^{(1)}\right\}$ and $\left\{x_{1}^{(2)}, \ldots, x_{q_{\ell}}^{(2)}\right\}$ to be $\mathbb{X}^{(1)}$ and $\mathbb{X}^{(2)}$, respectively, it leads to the full kernel KCCA. With the choice $\tilde{\mathbb{X}}^{(1)}$ and $\tilde{\mathbb{X}}^{(2)}$, it leads to the reduced kernel KCCA. We refer the reader to our earlier discussion on basis selection in Section 3. 


\section{A.2. Independence test}

The following proposition validates the use of the $\log$-likelihood-ratio-based association measure $r_{\log }^{\kappa}:=-\sum_{v} \log \left(1-\left(\rho_{v}^{\kappa}\right)^{2}\right)$ for independence test.

Proposition 3. Under the null hypothesis that $X^{(1)}$ and $X^{(2)}$ are independent, the sample version of $r_{\log }^{\kappa}$ based on systems (19) and (20) converges in distribution to $\chi_{\left(p_{\ell}-1\right)\left(q_{\ell}-1\right)}^{2}$, as $n \rightarrow \infty$.

Proof. As we have shown the systems are linear independent and are essentially dense, the asymptotic distribution follows from Proposition 5.3 of Dauxois and Nkiet. The only thing left to check is a scaling factor, $K_{r_{\log }}$, calculated as follows:

$$
K_{r_{\log }}=\left.\frac{\partial r_{\log }^{\kappa}}{\partial\left(\rho_{\ell}^{\kappa}\right)^{2}}\right|_{\rho_{\ell}^{\kappa}=0}=\left.\frac{1}{1-\left(\rho_{\ell}^{\kappa}\right)^{2}}\right|_{\rho_{\ell}^{\kappa}=0}=1 .
$$

Proof is completed.

Though we do not need any Gaussian assumption for the asymptotic $\chi^{2}$ distribution in Proposition 3, we would still like to point out the heuristic behind our test statistic for independence. In a study on kernel data transformation (Huang and Hwand, 2006), it is shown that low-dimensional projections of high-dimensional kernel data are approximately Gaussian. Thus, we use the Gaussian MLR-based test statistic instead of $r_{\mathrm{D} \& \mathrm{~N}}^{\mathrm{spl}}$-based test statistic (10) for independence.

In summary, the basic concept of KCCA is to develop parallel procedure as in LCCA for convenient nonlinear extension. The implementation of such a nonlinear generalization is done via using the parametric notion of the classical LCCA in an RKHS instead of in the pattern Euclidean space. Such a nonlinear extension using kernel framework is easy to implement compared with other nonlinear and nonparametric approaches.

\section{References}

Akaho, S., 2001. A kernel method for canonical correlation analysis. In: International Meeting of Psychometric Society (IMPS2001). Anderson, T.W., 2003. An Introduction to Multivariate Statistical Analysis. third ed. Wiley, New York. Asuncion, A., Newman, D.J., 2007. UCI repository of smachine learning databases http://www.ics.uci.edu/ mlearn/MLRepository.html. Bach, F.R., Jordan, M.I., 2002. Kernel independent component analysis. J. Mach. Learning Res. 3, 1-48. Bartlett, M.S., 1947a. Multivariate analysis. J. Roy. Statist. Soc. 9 (Suppl.), 176-197.

Bartlett, M.S., 1947b. The general canonical correlation distribution. Ann. Math. Statist. 18, 1-17.

Berlinet, A., Thomas-Agnan, C., 2004. Reproducing Kernel Hilbert Spaces in Probability and Statistics. Kluwer, Boston.

Cortes, C., Vapnik, V., 1995. Support vector networks. Mach. Learning 20, 273-279.

Cristianini, N., Shawe-Taylor, J., 2000. An Introduction to Support Vector Machines and Other Kernel-Based Learning Methods. Cambridge University Press, Cambridge, UK.

Dauxois, J., Nkiet, G.M., 1997. Canonical analysis of two Euclidean subspaces and its applications. Linear Algebra Appl. 264, $355-388$.

Dauxois, J., Nkiet, G.M., 1998. Nonlinear canonical analysis and independence tests. Ann. Statist. 26, 1254-1278.

Dauxois, J., Nkiet, G.M., 2002. Measure of association for Hilbert subspaces and some applications. J. Multivariate Anal. 82, $263-298$.

Dauxois, J., Romain, Y., Viguier, S., 1993. Comparison of two factor subspaces. J. Multivariate Anal. 44, 160-178.

Dauxois, J., Nkiet, G.M., Romain, Y., 2004. Canonical analysis relative to a closed subspace. Linear Algebra Appl. 388, 119-145

Eubank, R., Hsing, T., 2006. Canonical correlation for stochastic processes, preprint http://www.stat.osu.edu/hsing/papers/CCpaper-rev1.pdf.

Gretton, A., Herbrich, R., Smola, A., 2003. The kernel mutual information. Technical Report, MPI for Biological Cybernetics, Tuebingen, Germany.

Gretton, A., Herbrich, R., Smola, A., Bousquet, O., Schölkopf, B., 2005. Kernel methods for measuring independence. J. Mach. Learning Res. 6, 2075-2129.

Hardoon, D.R., Szedmak, S., Shawe-Taylor, J., 2004. Canonical correlation analysis: an overview with application to learning methods. Neural Comput. 16, 2639-2664.

Hastie, T., Tibshirani, R., Friedman, J., 2001. The Elements of Statistical Learning: Data Mining, Inference, and Prediction. Springer, New York.

Hotelling, H., 1936. Relations between two sets of variates. Biometrika 28, 321-377.

Hsing, T., Liu, L.Y., Brun, M., Dougherty, E.R., 2005. The coefficient of intrinsic dependence. Pattern Recognition 38, 623-636.

Huang, C.M., Lee, Y.J., Lin, D.K.J., Huang, S.Y., 2007. Model selection for support vector machines via uniform design. A special issue on machine learning and robust data mining. Comput. Statist. Data Anal. 52, 335-346.

Huang, S.Y., Hwand, C.R., 2006. Kernel Fisher discriminant analysis in Gaussian reproducing kernel Hilbert spaces-theory. Technical Report, Institute of Statistical Science, Academia Sinica http://www.stat.sinica.edu.tw/syhuang/.

Jensen, D.R., Mayer, L.S., 1977. Some variational results and their applications in multiple inference. Ann. Statist. 5, 922-931.

Kuss, M., Graepel, T., 2003. The geometry of kernel canonical correlation analysis. Technical Report, Max Planck Institute for Biological Cybernetics, Germany.

Lee, Y.J., Huang, S.Y., 2007. Reduced support vector machines: a statistical theory. IEEE Trans. Neural Networks $18,1-13$.

Lee, Y.J., Mangasarian, O.L., 2001. RSVM: reduced support vector machines. In: Proceeding of the First International Conference on Data Mining, SIAM.

Ramsay, J.O., Silverman, B.W., 2005. Functional Data Analysis. second ed. Springer, New York.

Schölkopf, B., Smola, A., 2002. Learning with Kernels: Support Vector Machines, Regularization, Optimization, and Beyond. MIT Press, Cambridge, MA

Smola, A., Schölkopf, B., 2000. Sparse greedy matrix approximation for machine learning. In: Proceedings of the 17th International Conference on Machine Learning. Morgan Kaufmann, San Francisco, CA, pp. 911-918.

Snelson, E., Ghahramani, Z., 2005. Sparse Gaussian processes using pseudo-inputs. In: Weiss, Y., Schölkopf, B., Platt, J. (Eds.), Advances in Neural Information Processing Systems, vol. 18. MIT Press, Cambridge, MA.

Vapnik, V.N., 1998. Statistical Learning Theory. Wiley, New York.

Wang, J., Neskovic, P., Cooper, L.N., 2005. Training data selection for support vector machines. In: Wang, L., Chen, K., Ong, Y.-S. (Eds.), Advances in Natural Computation: Proceedings, Part I, First International Conference. Lecture Notes in Computer Science, vol. 3610. Springer, Berlin, pp. 554-564.

Williams, C.K.I., Seeger, M., 2001. Using the Nyström method to speed up kernel machines. In: Leen, T.K., Dietterich, T.G., Tresp, V. (Eds.), Advances in Neural Information Processing Systems, vol. 13. MIT Press, Cambridge, MA, pp. 682-688. 